\title{
A SPECIALISED CONTINUED FRACTION
}

\author{
A. J. VAN DER POORTEN ${ }^{1}$ AND J. Shallit ${ }^{2}$ \\ Macquarie University and Waterloo
}

\begin{abstract}
We display a number with a surprising continued fraction expansion and show that we may explain that expansion as a specialisation of the continued fraction expansion of a formal series: A series $\sum c_{h} X^{-h}$ has a continued fraction expansion with partial quotients polynomials in $X$ of positive degree (other, perhaps than the 0 -th partial quotient). Simple arguments, let alone examples, demonstrate that it is noteworthy if those partial quotients happen to have rational integer coefficients only. In that special case one may replace the variable $X$ by an integer $\geq 2$; that is: one may 'specialise' and thereby proceed to obtain the regular continued fraction expansion of values of the series. And that is significant because, generally, it is difficult to obtain the explicit continued fraction expansion of a number presented in different shape. Our example leads to a series with a specialisable continued fraction expansion and, a little surprisingly, our arguments suggest that the phenomenon of specialisability for series of the kind appearing here may be reserved to just the special subclass of series we happen to have stumbled upon.
\end{abstract}

\section{INTRODUCTION}

A dozen or so years ago, one of us, heavily influenced by the cult of Fibonacci, noticed the continued fraction expansion

$$
\begin{aligned}
& \text { (1) } 2^{-1}+2^{-2}+2^{-3}+2^{-5}+\cdots+2^{-F_{h}}+\cdots \\
& =[0,1,10,6,1,6,2,14,4,124,2,1,2,2039,1,9,1,1,1,262111,2,8, \\
& \quad 1,1,1,3,1,536870655,4,16,3,1,3,7,1,140737488347135, \ldots] .
\end{aligned}
$$

The increasing sequence of very large partial quotients demands explanation; truncations of the sum do not yield convergents and the shape of the very good approximations is not immediately obvious. We show here that a correct context for the cited expansion can be discovered in remarks of Mendès France and van der Poorten [6], wherein one considers continued fractions of formal Laurent series

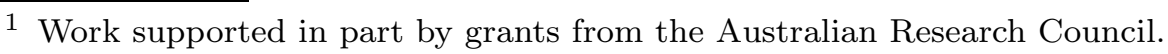

2 Supported in part by a grant from NSERC Canada.
}

1991 Mathematics subject classification: 11A55, 11Y65, 11J70 
and then specialises the variable to an appropriate integer. Indeed, we found the arguments detailed in [4] and at that time noticed experimentally that

(2) $X^{-1}+X^{-2}+X^{-3}+X^{-5}+\cdots+X^{-F_{h}}+\ldots$

$$
\begin{gathered}
=\left[0, X-1, X^{2}+2 X+2, X^{3}-X^{2}+2 X-1,-X^{3}+X-1,-X,-X^{4}+X,-X^{2},\right. \\
-X^{7}+X^{2},-X-1, X^{2}-X+1, X^{11}-X^{3},-X^{3}-X,-X, X, X^{18}-X^{5}, \\
\left.-X, X^{3}+1, X,-X,-X-1,-X+1,-X^{29}+X^{8}, X-1, \ldots\right] .
\end{gathered}
$$

The limited number of shapes for the partial quotients, the phenomenon of selfsimilarity whereby bits and pieces from early in the sequence of partial quotients reappear subsequently, and of most importance the fact that all of the partial quotients have rational integer coefficients, all demand explanation and generalisation. We provide that here.

Above, and in the sequel, $\left(F_{h}\right)$ denotes the sequence of Fibonacci numbers defined by the recurrence relation $F_{h+2}=F_{h+1}+F_{h}$ and the initial values $F_{0}=0, F_{1}=1$.

Not too surprisingly, the phenomenon (1) has been noticed by others; our correspondence contains a letter of March, 1989 from M. J. Knight enquiring about the continued fraction of $\sum_{n \geq 1} B^{-F_{n}}$.

\section{FIRST PRINCIPLES}

Our viewpoint is formal. A continued fraction is of course an expression of the shape

$$
a_{0}+\frac{1}{a_{1}}+\frac{1}{a_{2}}+\frac{1}{a_{3}}+\frac{}{\ddots}
$$

which we denote in a space-saving flat notation by

$$
\left[a_{0}, a_{1}, a_{2}, a_{3}, \ldots \ldots\right]
$$

Everything follows from the correspondence whereby one has

$$
\left(\begin{array}{cc}
a_{0} & 1 \\
1 & 0
\end{array}\right)\left(\begin{array}{cc}
a_{1} & 1 \\
1 & 0
\end{array}\right) \cdots \cdots\left(\begin{array}{cc}
a_{n} & 1 \\
1 & 0
\end{array}\right)=\left(\begin{array}{cc}
p_{n} & p_{n-1} \\
q_{n} & q_{n-1}
\end{array}\right) \text { for } n=0,1,2, \ldots \ldots
$$

if and only if

$$
\frac{p_{n}}{q_{n}}=\left[a_{0}, a_{1}, \ldots \ldots, a_{n}\right] \text { for } n=0,1,2, \ldots \ldots,
$$

Thus, for example, taking the transpose in the correspondence we see that

$$
\left[a_{n}, a_{n-1}, \ldots, a_{1}\right]=\frac{q_{n}}{q_{n-1}}
$$


and, taking determinants, that

$$
p_{n} q_{n-1}-p_{n-1} q_{n}=(-1)^{n+1} \text { so } \frac{p_{n}}{q_{n}}=\frac{p_{n-1}}{q_{n-1}}+(-1)^{n-1} \frac{1}{q_{n-1} q_{n}} .
$$

From this last observation once sees easily that if the partial quotients $a_{h}$ are polynomials of degree at least 1 then the convergents $p_{h} / q_{h}$ converge to a formal series in $X^{-1}$. These remarks are given in [6].

The regular continued fraction expansion of a real number has partial quotients $a_{h}$ that are positive integers (other than perhaps for $a_{0}$ which may take any integer value); zero, negative and fractional partial quotients are termed inadmissible. Similarly, the admissible partial quotients $a_{h}$ of a formal series in $X^{-1}$ are polynomials of degree at least 1 (except perhaps for $a_{0}$ ).

We can readily see the following invaluable lemma:

\section{Folding Lemma.}

$$
\frac{p_{n}}{q_{n}}+\frac{(-1)^{n}}{x q_{n}^{2}}=\left[a_{0}, \vec{w}, x-\frac{q_{n-1}}{q_{n}}\right]=\left[a_{0}, \vec{w}, x,-\overleftarrow{w}\right]
$$

Here $\vec{w}$ is a convenient abbreviation for the word $a_{1}, a_{2}, \ldots, a_{n}$ and, accordingly, $-\overleftarrow{w}$ denotes the word $-a_{n},-a_{n-1}, \ldots,-a_{1}$

For a proof, see [5] or [8].

The name of the lemma comes from the observation that its iterated application leads to a pattern of signs corresponding to the creases in a sheet of paper repeatedly folded in half; for details, see [8].

\section{Our Program}

We shall show that the continued fraction expansion of the series $X^{-1}+X^{-2}+$ $X^{-3}+X^{-5}+\cdots+X^{-F_{h}}+\ldots$ indeed has partial quotients of limited shapes and that all have integer coefficients. Then we specialise by replacing $X$ by an integer $x$ with $|x| \geq 2$, obtaining a convergent continued fraction with integral partial quotients. Of course, there may well be negative or zero partial quotients. But it is easily verified that

$$
[\ldots, a, 0, b, \ldots]=[\ldots, a+b, \ldots]
$$

and

$$
[\ldots, a,-\beta]=[\ldots, a, 0,-1,1,-1,0, \beta]=[\ldots, a-1,1, \beta-1]
$$

this yields a technique for rendering negative partial quotients positive. The case $\beta=1$ will not arise below; but it is easy to verify that $[\ldots, a-1,1,0]=$ $[\ldots, a-1]$, and clearly $[\ldots, a,-1]=[\ldots, a-1]$. 


\section{Calculations and Guesses}

Set $s_{n}=X^{-1}+X^{-2}+X^{-3}+X^{-5}+\cdots+X^{-F_{n}}$. Computation reveals that

$$
\begin{aligned}
s_{2}= & {[0, X], } \\
s_{3}= & {[0, X-1, X+1], } \\
s_{4}= & {\left[0, X-1, X^{2}+X+1\right], } \\
s_{5}= & {\left[0, X-1, X^{2}+2 X+2,-X^{2}+X-1\right], } \\
s_{6}= & {\left[0, X-1, X^{2}+2 X+2, X^{3}-X^{2}+2 X-1, X,-X\right], } \\
s_{7}= & {\left[0, X-1, X^{2}+2 X+2, X^{3}-X^{2}+2 X-1,-X^{3}+X-1,-X, X, X+1, X-1\right], } \\
s_{8}= & {\left[0, X-1, X^{2}+2 X+2, X^{3}-X^{2}+2 X-1,-X^{3}+X-1,-X,-X^{4}+X,-X^{2},\right.} \\
& \left.X^{2}, X^{2}+X+1, X-1\right], \\
s_{9}= & {\left[0, X-1, X^{2}+2 X+2, X^{3}-X^{2}+2 X-1,-X^{3}+X-1,-X,-X^{4}+X,-X^{2},\right.} \\
- & \left.X^{7}+X^{2},-X-1, X^{2}-X+1,-X^{3},-X^{2}+X-1, X^{2}+2 X+2, X-1\right], \\
s_{10}= & {\left[0, X-1, X^{2}+2 X+2, X^{3}-X^{2}+2 X-1,-X^{3}+X-1,-X,-X^{4}+X,-X^{2},\right.} \\
- & X^{7}+X^{2},-X-1, X^{2}-X+1, X^{11}-X^{3},-X^{3}-X,-X, X,-X^{5}, \\
- & \left.X, X, X^{3}-X^{2}+2 X-1, X^{2}+2 X+2, X-1\right], \\
s_{11}= & {\left[0, X-1, X^{2}+2 X+2, X^{3}-X^{2}+2 X-1,-X^{3}+X-1,-X,-X^{4}+X,-X^{2},\right.} \\
& -X^{7}+X^{2},-X-1, X^{2}-X+1, X^{11}-X^{3},-X^{3}-X,-X, X, X^{18}-X^{5}, \\
& -X, X^{3}+1, X,-X,-X-1,-X+1, X^{8}, X-1, X+1, X, \\
& \left.-X,-X^{3}+X-1, X^{3}-X^{2}+2 X-1, X^{2}+2 X+2, X-1\right] .
\end{aligned}
$$

These, and yet more extended calculations which we eschew reporting, suggest a symmetry in the expansion of $s_{h}$ and that suggests employing the lemma and induction. Accordingly we set $s_{h}=\left[0, f_{h}\right]$ with the word $f_{h}$ assumed to be of even length (as indeed it appears to be for $h \geq 7$ ). The general theory entails that $s_{h}=\left[0, f_{h}\right]=p / q$ with the polynomial $q$ a constant multiple of $X^{F_{h}}$. Again the data suggests the assumption that $q= \pm X^{F_{h}}$ (in fact $+X^{F_{h}}$ for $h \geq 7$ ). With this assumption, noting that $F_{h+1}-2 F_{h}=-F_{h-2}$,

$$
s_{h+1}=s_{h}+X^{-F_{h+1}}=\frac{p}{q}+\frac{1}{X^{-F_{h-2} q^{2}}}
$$

and the lemma yields

$$
s_{h+1}=\left[0, f_{h}, X^{-F_{h-2}},-\overleftarrow{f_{h}}\right]
$$

with a nastily inadmissible partial quotient $X^{-F_{h-2}}$.

At this point, one might be tempted to give up and apply the methods in [1]. However, in hindsight, it is better to stay with the result provided directly by the Folding Lemma, namely

$$
s_{h+1}=s_{h}+X^{-F_{h+1}}=\frac{p}{q}+\frac{1}{X^{-F_{h-2}} q^{2}}=\left[0, f_{h}, X^{-F_{h-2}}-q^{\prime} / q\right] .
$$


Here $q^{\prime}$ denotes the denominator of the next to last convergent to $s_{h}$ and, as already remarked, we may take $q= \pm X^{F_{h}}$. This approach succeeds because $q^{\prime}$ has a particularly simple and congenial shape which, moreover, is readily found.

\section{The Main Result}

We now state the main result of this paper:

Theorem 1. Let $\left(F_{h}\right)$ be the sequence of Fibonacci numbers defined by the recurrence relation $F_{h+2}=F_{h+1}+F_{h}$ and initial conditions $F_{0}=0, F_{1}=1$. Set

$$
s_{h}=X^{-1}+X^{-2}+X^{-3}+X^{-5}+\cdots+X^{-F_{h}}=\left[0, f_{h}\right] .
$$

Then the words $f_{h}, 2 \leq h \leq 11$, are given by $s_{h}=\left[0, f_{h}\right]$ as listed in $\S 4$. Let $g_{h}$ be the word

$$
g_{h}=f_{h-1}, 0,-f_{h-5},-X^{L_{h-5}}, \overleftarrow{f_{h-5}}, 0,-f_{h-4}
$$

Then for $h \geq 11$

$$
\begin{aligned}
& s_{h+1}=\left[0, f_{h}, 0,-f_{h-4},-X^{L_{h-4}}, \overleftarrow{f_{h-4}}, 0,-f_{h-3}, X^{F_{h-4}}, \overleftarrow{f_{h-3}}\right] \\
& =\left[0, g_{h}, X^{F_{h-5}}, \overleftarrow{f_{h-4}}, 0,-f_{h-4},-X^{L_{h-4}}, \overleftarrow{f_{h-4}}, 0,-f_{h-3}, X^{F_{h-4}}, \overleftarrow{f_{h-3}}\right] \\
= & {\left[0, g_{h}, X^{F_{h-5}}-X^{L_{h-4}}, \overleftarrow{f_{h-4}}, 0,-f_{h-3}, X^{F_{h-4}}, \overleftarrow{f_{h-3}}\right]=\left[0, g_{h+1}, X^{F_{h-4}}, \overleftarrow{f_{h-3}}\right] }
\end{aligned}
$$

and

$$
s_{\infty}=X^{-1}+X^{-2}+X^{-3}+X^{-5}+\cdots=\lim _{h \rightarrow \infty}\left[0, g_{h}\right]
$$

Proof. The proof is by induction on $h$. The base case is $h=11$. Our induction hypothesis also includes the assumption that $s_{h}=\left[0, f_{h}\right]$ has $f_{h}$ of even length for $h \geq 7$. This can easily be verified for $7 \leq h \leq 11$ by examining the computations in $\S 4$, and its truth in general will follow from our computations below.

We notice that $q^{\prime}$ is that polynomial of degree less than $F_{h}$ satisfying $p q^{\prime}-p^{\prime} q=$ -1 , with $p^{\prime}$ a polynomial also of degree less than $F_{h}$. Here we apply our convention whereby $p / q$ denotes the last partial quotient and $p^{\prime} / q^{\prime}$ the penultimate partial quotient. On dividing by $q^{2}$, this is

$$
s_{h}\left(q^{\prime} / q\right)=-1 / X^{2 F_{h}}+\left(p^{\prime} / q\right)
$$

But

$$
s_{h}\left(s_{h}-X^{-F_{h-3}}-2 X^{-F_{h}}\right)
$$

is of the shape $(7)$ by virtue of the fact that the sequence $\left(F_{h}\right)$ is strictly increasing with $F_{h-2}+F_{h-1} \leq F_{h}$ and $2 F_{h-1}=F_{h-3}+F_{h}$. Indeed, the inequality (actually an equality for the sequence $\left.\left(F_{h}\right)\right)$ entails that in $s_{h}\left(s_{h}-2 X^{-F_{h}}\right)$ the only terms of degree less than $-F_{h}$ are $-X^{-2 F_{h}}$ as required, and $X^{-2 F_{h-1}}$. Subtracting $X^{-F_{h-3}}$ 
from $s_{h}-2 X^{-F_{h}}$ yields the additional term $-X^{-F_{h-3}-F_{h}}$ of degree less than $-F_{h}$ which, by the recurrence relation just cited, cancels the offending term. Thus

$$
q^{\prime} / q=s_{h}-X^{-F_{h-3}}-2 X^{-F_{h}} .
$$

Of course it happened that we noticed this from our experimental evidence, but, in hindsight, it is apparent that $q^{\prime} / q$ can readily be found without such guidance.

Combining (8) with (6) we get

$$
\begin{aligned}
& s_{h+1}=\left[0, f_{h}, X^{-F_{h-2}}-q^{\prime} / q\right] \\
= & {\left[0, f_{h},-\left(s_{h}-X^{-F_{h-3}}-2 X^{-F_{h}}-X^{-F_{h-2}}\right)\right]=\left[0, f_{h},-\left(s_{h-4}+X^{-F_{h-1}}-X^{-F_{h}}\right)\right] . }
\end{aligned}
$$

So all we need do is determine the continued fraction expansion of

$$
-\left(s_{h-4}+X^{-F_{h-1}}-X^{-F_{h}}\right) .
$$

If $h \geq 11$, then by induction, $F_{h-1}-2 F_{h-4}=F_{h-3}+F_{h-5}$, and the lemma,

$$
s_{h-4}+X^{-F_{h-1}}=\left[0, f_{h-4}, X^{L_{h-4}},-\overleftarrow{f_{h-4}}\right]
$$

here, as is traditional, $\left(L_{h}\right)$ denotes the sequence of Lucas numbers defined by $L_{0}=2, L_{1}=1$ and $L_{h+2}=L_{h+1}+L_{h}$ (that is, $L_{h+1}=F_{h}+F_{h+2}$ ). Finally, using the folding lemma again, we can add $-X^{-F_{h}}$ to $s_{h-4}+X^{-F_{h-1}}$ and obtain the expansion of $s_{h-4}+X^{-F_{h-1}}-X^{-F_{h}}$ by noting that $F_{h}-2 F_{h-1}=-F_{h-3}$ and according to (6) appending the partial quotient $X^{-F_{h-3}}-q^{\prime} / q$. That is,

$$
s_{h-4}+X^{-F_{h-1}}-X^{-F_{h}}=\left[0, f_{h-4}, X^{L_{h-4}},-\overleftarrow{f_{h-4}}, X^{-F_{h-3}}-q^{\prime} / q\right]
$$

Here, $q^{\prime} / q$ refers to $(9)$ in which the word following the 0 -th partial quotient is of odd length, $q=X^{F_{h-1}}$, and we find $q^{\prime} / q$ in virtue of

$$
\left(s_{h-4}+X^{-F_{h-1}}\right)\left(-s_{h-4}+X^{-F_{h-1}}\right)=X^{-2 F_{h-1}}+p^{\prime} / X^{F_{h-1}}
$$

having the appropriate shape (7). We have

$$
\begin{aligned}
& X^{-F_{h-3}}-q^{\prime} / q=s_{h-4}-X^{-F_{h-1}}+X^{-F_{h-3}} \\
& =s_{h-3}-X^{-F_{h-1}}=\left[0, f_{h-3},-X^{F_{h-4}},-\overleftarrow{f_{h-3}}\right],
\end{aligned}
$$

where we have used $F_{h-1}-2 F_{h-3}=F_{h-4}$, the lemma and the fact that $h \geq 11$. Thus

$$
s_{h-4}+X^{-F_{h-1}}-X^{-F_{h}}=\left[0, f_{h-4}, X^{L_{h-4}},-\overleftarrow{f_{h-4}}, 0, f_{h-3},-X^{F_{h-4}},-\overleftarrow{f_{h-3}}\right] .
$$

So our inductive assumptions entail for $h \geq 11$ that

$$
s_{h+1}=\left[0, f_{h}, 0,-f_{h-4},-X^{L_{h-4}}, \overleftarrow{f_{h-4}}, 0,-f_{h-3}, X^{F_{h-4}}, \overleftarrow{f_{h-3}}\right]
$$


and the principal remaining problem remains to check the initial conditions. It suffices to note the computations at the beginning of $\S 4$.

To complete the proof, we apply (10) to replace $f_{h}$ by

$$
f_{h-1}, 0,-f_{h-5},-X^{L_{h-5}}, \overleftarrow{f_{h-5}}, 0,-f_{h-4}, X^{F_{h-5}}, \overleftarrow{f_{h-4}}
$$

to explicitly reveal the partial quotients $X^{F_{h-5}}-X^{L_{h-4}}$. Of course it suffices to report that $s_{\infty}$ is the direct limit of the expansions $\left[0, f_{h}\right]$ but it remains useful to notice that it is the prefix $g_{h}$ of $f_{h}$ that persists, and that it is given by

$$
g_{h+1}=g_{h}, X^{F_{h-5}}-X^{L_{h-4}}, \overleftarrow{f_{h-4}}, 0,-f_{h-3}
$$

Finally, we note that from Eqs. (4) and (10), it follows that $f_{h+1}$ has even length.

\section{Corollaries to the Main Result}

Our main result allows us to explicitly characterise the partial quotients in the continued fraction for $s_{\infty}$ :

Corollary 1.1. A polynomial $a$ is a partial quotient in the expansion of $s_{\infty}$ if and only if $a$ or $-a$ occurs in the following list: $X+1 ; X^{2} \pm X+1 ; X^{2}+2 X+2 ; X^{3}+$ $1 ; X^{3}+X ; X^{3}-X+1 ; X^{3}-X^{2}+2 X-1 ; X^{F_{n}} ; X^{L_{n+2}} ; X^{L_{n+2}}-X^{F_{n+1}}$, for $n \geq$ 1 .

Corollary 1.2. The large partial quotients

2039, 262111, 536870655, 140737488347135, 75557863725914321321983, . .

in the continued fraction expansion of $2^{-1}+2^{-2}+2^{-3}+2^{-5}+\cdots$ differ by 1 from the numbers $2^{L_{h+1}}-2^{F_{h}}$, for $h \geq 4$.

Proof. These partial quotients of course arise from the partial quotients $X^{F_{h-5}}-$ $X^{L_{h-4}}$ after specialising $X$ to 2 . Now rendering the specialised expansion admissible (by making all partial quotients positive) yields the observed partial quotients.

Theorem 1 allows us to give a new proof of the following result (see [4]), a new explicit irrationality measure:

Proposition 1.3. The sum $2^{-1}+2^{-2}+2^{-3}+2^{-5}+\cdots$ converges to a transcendental number.

Proof. Our remarks at Eq. (7) entail that

$$
\begin{aligned}
& \left(X^{-F_{h-2}}+X^{-F_{h-1}}-X^{-F_{h}}\right) s_{\infty}=\text { terms of degree } \geq-F_{h}-F_{h-4} \\
& +X^{-F_{h-1}-F_{h+1}}+\text { terms of yet lower degree. }
\end{aligned}
$$


The content of this observation is that there is no term of degree $-2 F_{h-1}$ because $2 F_{h-1}=F_{h}+F_{h-3}$ and no term of degree $-F_{h-2}-F_{h+1}$ because $F_{h-2}+F_{h+1}=$ $2 F_{h}$; and of course $F_{h-2}+F_{h-1}=F_{h}$.

Accordingly, we set $q_{h}=q_{h}(X)=X^{F_{h}+F_{h-4}}\left(X^{-F_{h-2}}+X^{-F_{h-1}}-X^{-F_{h}}\right)$ and note that $\operatorname{deg} q_{h}=F_{h}+F_{h-4}-F_{h-2}=F_{h-1}+F_{h-4}$. Then (11) asserts that there is a polynomial $p_{h}$ in the variable $X$ so that

$$
\operatorname{deg}\left(q_{h} s_{\infty}-p_{h}\right)=-\left(F_{h-1}+F_{h+1}-F_{h}-F_{h-4}\right)=-\operatorname{deg} q_{h}-\left(F_{h-3}+F_{h-5}\right) .
$$

By easy arguments detailed in [6], it follows that $p_{h} / q_{h}$ is a convergent to $s_{\infty}$ and that the next partial quotient has degree $F_{h-3}+F_{h-5}$.

Set $\phi=(1+\sqrt{5}) / 2$ and note that as $h \rightarrow \infty$

$$
\left(F_{h-3}+F_{h-5}\right) /\left(F_{h-1}+F_{h-4}\right) \rightarrow\left(\phi^{h-3}+\phi^{h-5}\right) /\left(\phi^{h-1}+\phi^{h-4}\right)=\left(\phi^{2}+1\right) /\left(\phi^{4}+\phi\right) \approx 0.427 .
$$

It follows that on specialising $X$ to 2 we have a sequence of rational approximations $p_{h}(2) / q_{h}(2)$ so that for sufficiently large $h$

$$
\left|2^{-1}+2^{-2}+2^{-3}+2^{-5}+\cdots-p_{h}(2) / q_{h}(2)\right|<q_{h}(2)^{-2.42},
$$

proving our assertion by Roth's Theorem [9].

Remark 1.4. On specialising $X$ to an integer $x$ with $|x| \geq 4$ one immediately has the nearest integer continued fraction expansion for the corresponding transcendental number.

Comment 1.5. The number $t_{h}$ for $h=0,1, \ldots$ of partial quotients in the word $f_{h+2}$ (after cancellations) happens to satisfy the recurrence relation

$$
t_{h+10}=2 t_{h+9}-t_{h+8}+2 t_{h+6}-4 t_{h+5}+2 t_{h+4}+2 t_{h+1}-2 t_{h},
$$

with initial conditions $1,2,2,3,5,8,10,14,20,30$. The characteristic polynomial of the recurrence sequence is

$$
(X-1)(X+1)\left(X^{8}-2 X^{7}+2 X^{6}-2 X^{5}+2 X^{3}-2 X^{2}+2 X-2\right),
$$

with dominant root $\lambda=1.3706175926 \ldots$. So the number of terms grows approximately like $\lambda^{h}$.

\section{Generalisations}

Careful inspection of our arguments suggests that the properties of the Fibonacci numbers actually used above are that the sequence $\left(F_{h}\right)$ is strictly increasing with $F_{h-2}+F_{h-1} \leq F_{h}$ and $2 F_{h-1}=F_{h-3}+F_{h}$, and of course that the initial partial quotients computed at $\S 6$ have integer coefficients. It follows immediately that, subject to the last condition — but it seems to be satisfied as soon as we choose an 
appropriate starting point for the sequence (in our example we started with $F_{2}$ ), our arguments apply to strictly increasing Lucas sequences generally.

Generally, it is plain that, at worst, any denominators that do occur amongst the coefficients of the partial quotients are composed of just finitely many different primes. In more formal terminology, the phenomenon that we have called specialisability is a matter of good reduction everywhere; whilst, more generally, one has good reduction almost everywhere. That is, our expansion in Theorem 1 makes sense for every finite base field $\mathbb{F}_{p}$. With an inappropriate starting point the expansion makes sense for almost every such base field. For example, starting with $F_{0}$ leads to bad reduction only at $p=2$.

A little more seems evident and we state it confidently as a conjecture.

Conjecture 2. Let $\left(T_{n}\right)$ be an increasing sequence of nonnegative integers satisfying a recurrence relation

$$
T_{h+d}=T_{h+d-1}+T_{h+d-2}+\cdots+T_{h} \text { with } d>1
$$

and set

$$
s_{n}=X^{-T_{d}}+X^{-T_{d+1}}+X^{-T_{d+2}}+\cdots+X^{-T_{n}} ; \quad s_{n}=\left[0, t_{n}\right] .
$$

Then, subject to appropriate initial conditions on the $T_{h}$, the words $t_{h}$ consist of polynomials with integer coefficients, which is to say that $s_{\infty}$ has a specialisable continued fraction expansion.

Remark. The point is that it is easy to see that we have $2 T_{h}=T_{h+1}+T_{h-d}$ and $T_{h-2}+T_{h-1} \leq T_{h}$. Moreover, our computations show that for small $d=$ $3,4,5,6, \ldots$ and initial values $0, \ldots, 0,1$ the commencing partial quotients are specialisable.

Before giving some additional vindication, including some details of the proof for the cases $d=3$ and $d=4$, we mention a related but independent proposition (cf. [4]).

Proposition 2.1. Let $x \geq 2$ be an integer. Then the numbers $x^{-T_{0}}+x^{-T_{1}}+$ $x^{-T_{2}}+\cdots$ are transcendental.

Proof. We see, by following the argument presented in Corollary 1.2, that the product

$$
\left(X^{-T_{h-d}}+X^{-T_{h-d+1}}+\cdots+X^{-T_{h-1}}-X^{-T_{h}}\right) s_{\infty}
$$

consists of terms with exponents $\geq-T_{h-d-2}-T_{h}$, there then is a gap, and the remaining terms have degree $\leq-T_{h-d+1}-T_{h+1}$.

Accordingly, we set

$$
q_{h}=q_{h}(X)=X^{T_{h}+T_{h-d-2}}\left(X^{-T_{h-d}}+\cdots+X^{-T_{h-1}}-X^{-T_{h}}\right)
$$


and note that $\operatorname{deg} q_{h}=T_{h}+T_{h-d-2}-T_{h-d}$. Thus there is a polynomial $p_{h}$ in the variable $X$ so that

$$
\begin{aligned}
& \operatorname{deg}\left(q_{h} s_{\infty}-p_{h}\right)=-\left(\left(T_{h-d+1}+T_{h+1}\right)-\left(T_{h}+T_{h-d-2}\right)\right) \\
= & -\operatorname{deg} q_{h}-\left(\left(T_{h-d+1}+T_{h+1}\right)-\left(2 T_{h-d-2}+2 T_{h}-T_{h-d}\right)\right)=-\operatorname{deg} q_{h}-\left(T_{h-d+1}-2 T_{h-d-2}\right) .
\end{aligned}
$$

Hence $p_{h} / q_{h}$ is a convergent to $s_{\infty}$ and the next partial quotient has degree $T_{h-d+1}-2 T_{h-d-2}$.

Let $\tau$ denote the unique zero of $X^{d}-X^{d-1}-\cdots-1$ outside the unit circle (cf. [7]), and note that as $h \rightarrow \infty$, we have

$$
\left(T_{h-d+1}-2 T_{h-d-2}\right) /\left(T_{h-d-2}+T_{h}-T_{h-d}\right) \rightarrow\left(\tau^{3}-2\right) /\left(\tau^{d+2}-\tau^{2}+1\right)=\theta>0 .
$$

It follows that on specialising $X$ to $x$ we have a sequence of rational approximations $p_{h}(x) / q_{h}(x)$ so that for sufficiently large $h$

$$
\left|x^{-T_{1}}+x^{-T_{2}}+x^{-T_{3}}+\cdots-p_{h}(x) / q_{h}(x)\right|<q_{h}(x)^{-(2+\theta)},
$$

proving our claim.

Remark. The size of $\tau$ can be conveniently estimated by $\tau^{d+1}-2 \tau^{d}+1=0$, so $2>\tau>2-\left(2^{d}-d / \tau\right)^{-1}$.

Evidence in support of the conjecture. We may suppose that $\left|t_{h}\right|$ is odd for all appropriately large $h$, for if not we can substitute $[\ldots, a]=[\ldots, a-1,1]$ and deal with the inadmissible partial quotient later by the rule $[\ldots, a, 1, \beta]=[\ldots, a+1,-\beta-1]$ as explained in $\S 3$. The cryptic notation below is that already explained and employed in $\S 6$. Thus to begin with we have

$$
s_{n+1}=s_{n}+X^{-T_{n+1}}=\left[0, t_{n},-X^{-T_{n-d}}-q^{\prime} / q\right]
$$

because $T_{n+1}-2 T_{n}=-T_{n-d}$. Moreover,

$$
-\left(s_{n-1}+X^{-T_{n}}\right)\left(s_{n-1}-X^{-T_{n}}-X^{-T_{n-d-1}}\right)=X^{-2 T_{n}}+p^{\prime} / q
$$

shows that we are next to append the continued fraction expansion of

$$
\begin{aligned}
-X^{-T_{n-d}}-q^{\prime} / q=s_{n-1}-X^{-T_{n}}-X^{-T_{n-d}}-X^{-T_{n-d-1}} & \\
& =s_{n-d-2}+X^{-T_{n-d+1}}+\cdots+X^{-T_{n-1}}-X^{-T_{n}} .
\end{aligned}
$$

But

$$
s_{n-d-2}+X^{-T_{n-d+1}}=\left[0, t_{n-d-2},-X^{T_{n-d+1}-2 T_{n-d-2}},-\overleftarrow{t_{n-d-2}}\right]
$$

that is, we apply the Folding Lemma explicitly. 
For large $d$ we now note that

$$
-\left(s_{n-d-2}+X^{-T_{n-d+1}}\right)\left(s_{n-d-2}-X^{-T_{n-d+1}}\right)=X^{-2 T_{n-d+1}}+p^{\prime} / q
$$

and $T_{n-d+2}-2 T_{n-d+1}=-T_{n-2 d+1}$ together entail that computing the continued fraction expansion of $s_{n-d-2}+X^{-T_{n-d+1}}+X^{-T_{n-d+2}}$ is a matter of appending the expansion of

$s_{n-d-2}-X^{-T_{n-d+1}}-X^{-T_{n-2 d+1}}=s_{n-2 d}+X^{-T_{n-2 d+2}}+\cdots+X^{-T_{n-d-2}}-X^{-T_{n-d+1}}$.

We need not slip ever deeper into a mire of increasing complexity as the case $d=3$ illustrates too amply. Indeed in that case the right hand side of (12) is just

$$
s_{n-5}-X^{-T_{n-2}}-X^{-T_{n-5}}=s_{n-6}-X^{-T_{n-2}}
$$

and its expansion is

$$
\left[0, t_{n-6}, X^{T_{n-2}-2 T_{n-6}},-\overleftarrow{t_{n-6}}\right]
$$

Then for $d=3$ it only remains to deal with subtracting $X^{-T_{n}}$. Fortunately, by

$$
-\left(s_{n-5}+X^{-T_{n-2}}+X^{-T_{n-1}}\right)\left(s_{n-6}+X^{-T_{n-2}}-X^{-T_{n-1}}\right)=X^{-2 T_{n-1}}+p^{\prime} / q,
$$

with the operative observation being that $2 T_{n-2}=T_{n-1}+T_{n-5}$, this is just a matter of appending the expansion of

$$
s_{n-6}+X^{-T_{n-2}}-X^{-T_{n-1}}+X^{-T_{n-4}},
$$

because $T_{n}-2 T_{n-1}=T_{n-4}$. But

$$
\begin{aligned}
s_{n-6}+X^{-T_{n-4}}+X^{-T_{n-2}=[0} & , t_{n-6},-X^{T_{n-4}-2 T_{n-6}},-\overleftarrow{t_{n-6}}, \\
& \left.-X^{T_{n-2}-2 T_{n-4}}, t_{n-6}, X^{T_{n-4}-2 T_{n-6}},-\overleftarrow{t_{n-6}}\right] .
\end{aligned}
$$

Now by

$$
-\left(s_{n-6}+X^{-T_{n-4}}+X^{-T_{n-2}}\right)\left(s_{n-6}+X^{-T_{n-4}}-X^{-T_{n-2}}\right)=X^{-2 T_{n-2}}+p^{\prime} / q
$$

and $T_{n-1}-2 T_{n-2}=T_{n-5}$, we have only to append the continued fraction expansion of

$$
s_{n-6}+X^{-T_{n-4}}-X^{-T_{n-2}}+X^{-T_{n-5}}=s_{n-4}-X^{-T_{n-2}} .
$$

When some dust has settled we see that for $d=3$

$$
\begin{aligned}
s_{n+1}= & {\left[0, t_{n}, 0, t_{n-5},-X^{T_{n-2}-2 T_{n-5}},-\overleftarrow{t_{n-5}}, 0, t_{n-6}, X^{T_{n-2}-2 T_{n-6}}\right.} \\
& -\overleftarrow{t_{n-6}}, 0, t_{n-6},-X^{T_{n-4}-2 T_{n-6}},-\overleftarrow{t_{n-6}},-X^{T_{n-2}-2 T_{n-4}}, \\
& \left.t_{n-6}, X^{T_{n-4}-2 T_{n-6}},-\overleftarrow{t_{n-6}}, 0, t_{n-4}, X^{T_{n-2}-2 T_{n-4}},-\overleftarrow{t_{n-4}}\right]
\end{aligned}
$$


The reader will notice an obvious cancellation as well as the practicability, much as in Theorem 1, of determining the portion of the expansion that remains invariant with increasing $n$. Yet more succinctly, when $d=4$ the right hand side of (12) is

$$
s_{n-8}+X^{-T_{n-6}}-X^{-T_{n-3}},
$$

which we expand by two folds. We note that

$$
-\left(s_{n-6}+X^{-T_{n-3}}+X^{-T_{n-2}}\right)\left(s_{n-6}-X^{-T_{n-7}}+X^{-T_{n-3}}-X^{-T_{n-2}}\right)=X^{-2 T_{n-2}}+p^{\prime} / q,
$$

whence adding $X^{-T_{n-1}}$ is a matter of appending the expansion of

$$
s_{n-6}-X^{-T_{n-7}}+X^{-T_{n-3}}-X^{-T_{n-2}}-X^{-T_{n-6}}=s_{n-8}+X^{-T_{n-3}}-X^{-T_{n-2}} .
$$

After appending one fold, that leads us to appending the continued fraction expansion of

$$
s_{n-8}+X^{-T_{n-3}}+X^{-T_{n-7}}=s_{n-7}+X^{-T_{n-3}},
$$

which is again just a fold. Subtracting $X^{-T_{n}}$ now turns out to be a matter of appending the expansion of

$$
\begin{aligned}
s_{n-6}-X^{-T_{n-6}}+X^{-T_{n-3}} & +X^{-T_{n-2}}-X^{-T_{n-1}}+X^{-T_{n-5}} \\
& =s_{n-7}+X^{-T_{n-5}}+X^{-T_{n-3}}+X^{-T_{n-2}}-X^{-T_{n-1}},
\end{aligned}
$$

which is of a level of complication with which we have already dealt. We do not trouble the printer with an explicit statement of the eventual result.

Bemusingly, the case of larger $d$ is rather different. The success of the program outlined above appears eventually to rely on the expansions of sums such as

$$
s_{k}+X^{-T_{k+2}}+\cdots+X^{-T_{k+d-2}} \quad k=1, \ldots, d-1
$$

being specialisable. So that we may glimpse this we give some cursory details of the case $d=5$. Here the right hand side of (12) is

$$
s_{n-10}+X^{-T_{n-8}}+X^{-T_{n-7}}-X^{-T_{n-4}}
$$

and when, after an easy fold, we attempt to add $X^{-T_{n-7}}$ we find ourselves endeavouring to expand

$$
s_{n-10}-X^{-T_{n-8}}-X^{-T_{n-13}}=s_{n-14}+X^{-T_{n-12}}+X^{-T_{n-11}}-X^{-T_{n-8}} .
$$

This is an 'and so on' which seems to terminate in the manner indicated above. 


\section{REMARKS AND REFLECTIONS}

The results given here are an interesting consequence of mathematical experimentation. The numerical observation (1) raised questions first answered by the experimental result (2). Ultimately Theorem 1 proves (2). En route we were led to experiments that suggest results as in Conjecture 2. There we prove (13) for the case $d=3$ and say enough to have proved the Conjecture for $d=4$. For the rest we give only a glimpse at, and less than a sketch of, a possible proof. Indeed, we are not entirely confident that we are recommending a viable program.

The pattern in (2) is only just vivid enough to be able to deduce something of the genre of Theorem 1 by eye. With increasing $d$ the experimental data is almost hopelessly complicated and the arguments suggested after Conjecture 2 become correspondingly the more instructive.

Whatever, it seems clear that Theorem 1 could not have been guessed and would not have been motivated without computer aid. The computations yielding (1) are almost infeasible by hand, and those yielding (2) are totally impractical without machine help.

Generally, on being shown (2) one might well guess the following: Suppose $\left(U_{h}\right)$ is a integer recurrence sequence, that is the solution of a linear homogeneous recurrence relation

$$
U_{h+n}=s_{1} U_{h+n-1}+\cdots+s_{n} U_{h} \quad h=0,1, \ldots,
$$

with integer coefficients $s_{1}, \ldots, s_{n}$ and integer initial values $U_{0}, \ldots, U_{n-1}$. Suppose further that the sequence $\left(U_{h}\right)$ is strictly increasing with $\lim _{h \rightarrow \infty} U_{h+1} / U_{h}=$ $\rho>1$. Then the series

$$
X^{-U_{0}}+X^{-U_{1}}+X^{-U_{2}}+\cdots
$$

has a specialisable continued fraction expansion.

With $\rho>2$ this is trivially true by the folding lemma, perhaps with the qualification that we must omit some initial terms of the series to ensure that always $U_{h+1} / U_{h} \geq$ 2 (and then $\rho=2$ will do); cf. $[2,3,10,11]$. However, when $1<\rho<2$ we have not as yet noticed any examples, except for the cases, relying on the identity $2 U_{h+n}=$ $U_{h+n+1}+U_{h}$, discussed here. We do not know what weight to give to our negative evidence. It is not utterly compelling because it is clear that any conjecture as above must be adjusted to allow the sequence to start with some term subsequent to $U_{0}$. On the other hand the evidence given in support of Conjecture 2 suggests that our present techniques are not up to constructing favourable examples, if there are any.

\section{ACKnowledgments}

We are grateful to the anonymous referee for his/her careful reading of the paper and several useful suggestions. 


\title{
10. Dedication
}

Whilst this paper was being completed my colleague and research assistant Ross Talent tragically died in a car accident. In the course of the 18 months that I worried about the problems settled here Ross helped by listening patiently. Ross shared an office with Jeff Shallit during Jeff's visit to Macquarie University when we finally learned how to explain the curious expansion (1). Ross would have read this manuscript and would have suggested how to avoid its remaining infelicities. I shall miss Ross grievously. AvdP

\section{REFERENCES}

[1] J.-P. Allouche, M. Mendès France and A. J. van der Poorten, An infinite product with bounded partial quotients, to appear in Acta Arithmetica.

[2] M. Kmošek, Rozwinięcie niektórych liczb niewymiernych na ułamki łańcuchowe, (Master's Thesis), Uniwersytet Warszawski, Warsaw, 1979.

[3] G. Köhler, Some more predictable continued fractions, Monatshefte Math. 89 (1980), 95-100.

[4] J. H. Loxton and A. J. van der Poorten, Arithmetic properties of certain functions in several variables III, Bull. Austral. Math. Soc. 16 (1977), 1547.

[5] M. Mendès France, Sur les fractions continues limitées, Acta Arithmetica 23 (1973), 207-215.

[6] M. Mendès France and A. J. van der Poorten, Some explicit continued fraction expansions, Mathematika 38 (1991), 1-9.

[7] E. P. Miles, Jr., Generalized Fibonacci numbers and associated matrices, Amer. Math. Monthly 67 (1960), 745-752.

[8] A. J. van der Poorten and J. Shallit, Folded continued fractions, J. Number Theory 40 (1992), 237-250.

[9] K. F. Roth, Rational approximations to algebraic numbers, Mathematika 2 (1955), 1-20; Corrigendum, p. 168.

[10] J. Shallit, Simple continued fractions for some irrational numbers, J. Number Theory 11 (1979), 209-217.

[11] J. Shallit, Simple continued fractions for some irrational numbers II, J. Number Theory 14 (1982), 228-231.

\author{
Alfred J. VAN DER PoOrten \\ Centre for Number Theory Research \\ MACQUARIE UNIVERSITY NSW 2109 \\ Australia \\ alf@macadam.mpce.mq.edu.au
}

JefFrey Shallit

Department of Computer Science 
UNIVERSITY OF WATERLOO

WAterloo, Ontario N2L 3G1

CANADA

shallit@graceland.waterloo.edu 